\title{
Design, Manufacture and Measurement of a Low-Cost Reflectarray for Global Earth Coverage
}

\author{
Daniel R. Prado, Adrián Campa, Manuel Arrebola, Marcos R. Pino, José A. Encinar, and Fernando Las-Heras
}

\begin{abstract}
In this work a low-cost reflectarray with isoflux pattern for global Earth coverage is designed, manufactured and measured at $30 \mathrm{GHz}$. The isoflux template is mathematically derived for a generic satellite and then particularized for a geostationary orbit. In order to reduce costs, the synthesis and design processes have to be accordingly adapted. First, the most simple unit cell is selected, consisting of a single layer of rectangular patches backed by a ground plane. This cell is not able to provide a $360^{\circ}$ phase range and thus the synthesis algorithm has to be modified to include a phase constraint to match the range provided by the element, ensuring at the same time a smooth variation of the elements in order to obtain a reliable design. This is further ensured by limiting the patch size to a given range during the design process. Finally, the reflectarray is manufactured and measured, showing good agreement with the simulations.
\end{abstract}

\section{Index Terms}

Reflectarray antenna, isoflux pattern, global coverage

\section{INTRODUCTION}

$\mathbf{R}$ EFLECTARRAY antennas combine the high gain and efficiency of parabolic reflectors with the low profile and flat nature of the arrays, at the expense of having an inherent lower bandwidth than the parabolic reflectors [1], although it may be overcome by using broadband topologies [2] or elements [3]. These features make the reflectarray a potential candidate for future space missions, since the costs involved in developing these antennas are lower than for shaped parabolic reflectors.

In this work, a reflectarray with an isoflux pattern for global Earth coverage is designed, manufactured and measured. The template for the isoflux pattern is mathematically developed for a generic satellite covering the whole Earth, and then is particularized for a geostationary orbit at $30 \mathrm{GHz}$. In order to reduce costs, a simple reflectarray comprised of only one layer of rectangular patches is designed. Rectangular patches are the simplest reflectarray unit cell, but they present the limitation of not being able to provide a full phase range of $360^{\circ}$ [1]. To overcome this limitation, the Intersection Approach (IA) algorithm [4] is used with phase constraints, limiting the allowable phase range during the synthesis process to that provided by the unit cell, while aiming at a smooth distribution of the patches after the design is made, which is further ensured by limiting the patch size range avoiding extreme values. These modifications in the synthesis and design processes allow to obtain a reliable shaped reflectarray design employing the simplest unit cell. Finally, simulations and measurements are compared showing good results.

\section{ISOFLUX PATTERN}

In Figure 1 a sketch of the Earth-satellite geometry is shown. There, $R_{e}$ is the radius of the Earth, $d_{\min }$ and $d_{\max }$ are minimum and maximum distances from the satellite to the Earth surface, and $\theta_{\max }$ the maximum coverage angle.

The goal of the isoflux pattern is to provide coverage with constant energy flux on the surface of the Earth. The attenuation curve is given by the Friis formula [5]

$$
A=20 \log (f)+20 \log (d)+32.5
$$

where $A$ is the attenuation given in $\mathrm{dB}, f$ is the frequency in $\mathrm{MHz}$ and $d$ is the distance from the satellite to the surface of the Earth in Km. If $\left(x_{s}, y_{s}\right)$ are the satellite coordinates and $\left(x_{e}, y_{e}\right)$ are the coordinates of a point in the Earth surface, $d$ is calculated as

$$
d=\sqrt{\left(x_{s}-x_{e}\right)^{2}+\left(y_{s}-y_{e}\right)^{2}} .
$$

The distance from the satellite to the surface of the Earth is given by its orbit, so $d_{\min }$ is known. Then, $d_{\max }$ is calculated using the Pythagorean theorem and $\theta_{\max }$ is obtained as

$$
\cos \theta_{\max }=\frac{d_{\max }}{d_{\min }+R_{e}}
$$

This work was supported in part by the European Space Agency (ESA) under contract ESTEC/AO/1-7064/12/NL/MH; by the Ministerio de Economía y Competitividad, under project TEC2014-54005-P (MIRIIEM); by the Gobierno del Principado de Asturias / FEDER under project GRUPIN14-114 and under grant BP12-063.

D. R. Prado, A. Campa, M. Arrebola, M. R. Pino and F. Las-Heras are with the Department of Electrical Engineering, Group of Signal Theory and Communications, Universidad de Oviedo (e-mail: drprado; arrebola; mpino; flasheras@tsc.uniovi.es).

Prof. J. A. Encinar is with Universidad Politécnica de Madrid (e-mail jose.encinar@upm.es). 


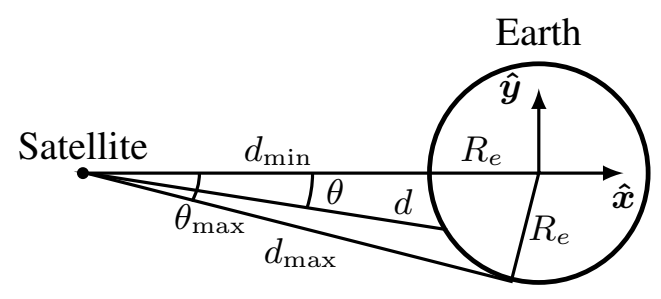

Figure 1: Sketch of the Earth-satellite geometry.

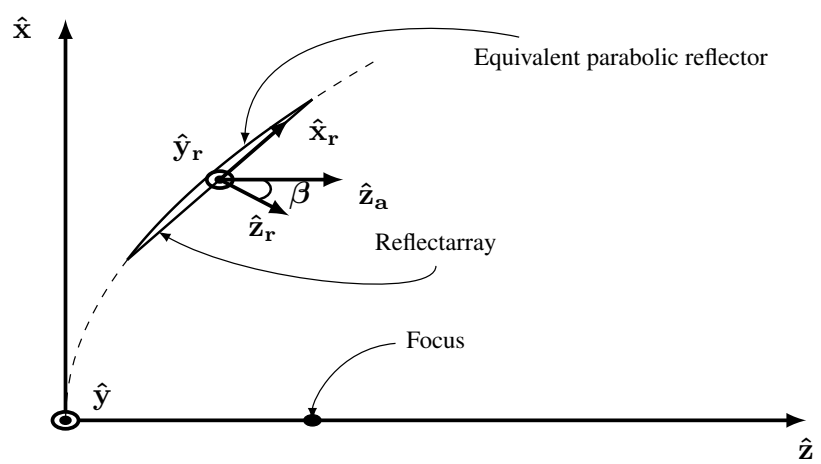

Figure 2: Reflectarray single-offset configuration.

Considering the origin of coordinates the center of the Earth, the points of its surface will follow the equation

$$
x^{2}+y^{2}=R_{e}^{2} .
$$

Then, the linear equation from the satellite to a point in the surface of the Earth can be expressed as

$$
y=m x+n,
$$

with $m=-\tan \theta$ and $n=-m\left(d_{\min }+R_{e}\right)$. Substituting (5) in (4), the following quadratic equation is obtained

$$
\left(1+m^{2}\right) x^{2}+2 m n x-\left(R_{e}^{2}-n^{2}\right)=0 .
$$

By solving (6) two solutions are obtained, which correspond to the intersection of the straight line $d$ with the Earth circumference. The one with $x<0$, according to Figure 1, is selected; and then $y$ is obtained with (4). This solution corresponds to the point $\left(x_{e}, y_{e}\right)$ in (2), which along with the known satellite position $\left(x_{s}, y_{s}\right)$ enable the calculation of the attenuation given by (1).

The attenuation curve is computed in a single plane, but since it is symmetric in $\varphi$, it is straightforward to obtain the attenuation in the whole Earth surface in spherical coordinates. It is common to perform reflectarray synthesis in the UV plane [1], knowing that $u=\sin \theta \cos \varphi$ and $v=\sin \theta \sin \varphi$.

The obtained attenuation pattern can be used directly as radiation pattern template if a symmetric antenna geometry is used. Apart from the attenuation curve, side lobe levels, ripple and a transition zone should also be specified. However, it is more common to use single-offset configurations [1], such as the one shown in Figure 2. The reflectarray radiates at an angle $\theta=\beta$ with regard to its own coordinate system, defined by $\left(\hat{x}_{r}, \hat{y}_{r}, \hat{z}_{r}\right)$. Hence, the centered templates have to be rotated by applying a proper transformation.

The template for the centered configuration in $(\theta, \varphi)$ coordinates is referenced to the reflectarray coordinate system $\left(\hat{x}_{r}, \hat{y}_{r}, \hat{z}_{r}\right)$ of Figure 2. Each point of the template is transformed from spherical coordinates to cartesian coordinates

$$
\begin{aligned}
x_{\mathrm{RA}} & =\sin \theta_{\mathrm{RA}} \cos \varphi_{\mathrm{RA}}, \\
y_{\mathrm{RA}} & =\sin \theta_{\mathrm{RA}} \sin \varphi_{\mathrm{RA}}, \\
z_{\mathrm{RA}} & =\cos \theta_{\mathrm{RA}},
\end{aligned}
$$

and then the following transformation is applied

$$
\left(\begin{array}{l}
x_{\mathrm{ANT}} \\
y_{\mathrm{ANT}} \\
z_{\mathrm{ANT}}
\end{array}\right)=\left(\begin{array}{ccc}
\cos \beta & 0 & \sin \beta \\
0 & 1 & 0 \\
-\sin \beta & 0 & \cos \beta
\end{array}\right)\left(\begin{array}{l}
x_{\mathrm{RA}} \\
y_{\mathrm{RA}} \\
z_{\mathrm{RA}}
\end{array}\right) .
$$

Then, the cartesian coordinates will be transformed to spherical coordinates and from there to UV coordinates, with the usual operations. 


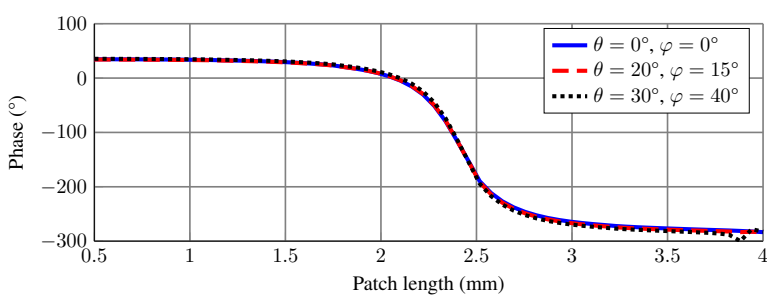

(a)

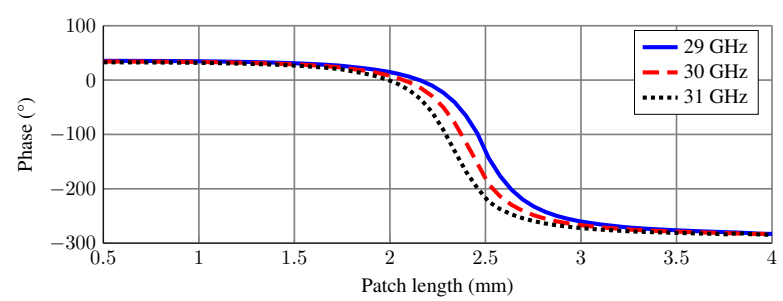

(b)

Figure 3: Phase range of the unit cell as a function of patch length for a periodicity of $0.4 \lambda$ at $30 \mathrm{GHz}$. (a) For different incidence angles at $30 \mathrm{GHz}$. (b) For different frequencies at $(\theta, \varphi)=\left(20^{\circ}, 15^{\circ}\right)$.

\section{ISOFLUX PATTERN SYNTHESIS}

\section{A. Antenna specifications}

The reflectarray is made up of 1528 elements disposed in a regular circular grid of $44 \times 44$ cells. The working frequency is $30 \mathrm{GHz}$ and the periodicity is $4 \mathrm{~mm} \times 4 \mathrm{~mm}(0.4 \lambda)$. The reflectarray is fed by a horn antenna whose phase center is placed at $(-40,0,195) \mathrm{mm}$ with regard to the reflectarray coordinate system (see Figure 2). The feed is modeled as a $\cos ^{q} \theta$ function following [6] with a $q$-factor of 14.8 , which produces an illumination taper of $-12 \mathrm{~dB}$. The requirements for the isoflux pattern are specified by a reflectarray with $\beta=20^{\circ}$, side lobe level of $-19 \mathrm{~dB}$ and $0.25 \mathrm{~dB}$ of allowable ripple, placed on a satellite in geostationary orbit.

\section{B. Unit cell study}

The reflectarray chosen element is the rectangular microstrip patch backed by a ground plane [7]. Since only a single layer is used, the provided phase range of the cell will be lower than $360^{\circ}$ [1]. Hence, it is important to study the element in order to delimit the phase range as a function of the patch size.

The Arlon $25 \mathrm{~N}$ substrate is selected, with a relative permittivity $\varepsilon_{r}=3.38$, thickness $h=18$ mil and loss $\operatorname{tangent} \tan \delta=$ 0.0025 at $10 \mathrm{GHz}$. Figure 3 shows the phase variation of the unit cell as a function of the patch length for different angles of incidence and frequencies. In this study, the patches are squares. The results show that, in this particular case, the incidence angle barely affects the phase shift introduced by the element. When the frequency is shifted, the phase variation is non-linear, since for dimensions lower than $2 \mathrm{~mm}$ and higher than $3 \mathrm{~mm}$ it barely changes, but between 2 and $3 \mathrm{~mm}$ the phase is shifted with frequency.

In order to maximize the available phase range avoiding extreme values of the patch lengths (which would compromise the local periodicity assumption when carrying out the design [1]), the patch length is restricted to vary in the range 1.7 to $3.2 \mathrm{~mm}$, which provides a phase range of about $300^{\circ}$.

\section{Pattern synthesis and design}

The reflectarray synthesis is carried out with the IA algorithm in several stages as is described in [4], starting with a high value of $q$ in order to artificially reduce the number of variables. Each step of the synthesis reduces the $q$-factor until its value reaches that of the horn feed. The starting point is a pencil beam pattern pointing at $(\theta, \varphi)=\left(20^{\circ}, 0^{\circ}\right)$. Also, since the generation of the isoflux template is parameterized, in the first steps of the synthesis the allowed ripple will be higher in order to facilitate convergence, and as the algorithm is approaching the solution, the ripple will be gradually reduced until the prescribed template value is achieved.

Since the selected unit cell only provides a phase range of $300^{\circ}$, the IA needs to be modified in order to impose a phase range restriction. In the general framework of the IA applied to array synthesis, the reflectarray is a particular case in which the amplitude is fixed by the incident field of the feed, and now the phase is also constrained in order to match the available phase range from the unit cell, allowing to vary only from $0^{\circ}$ to $-300^{\circ}$ to match the provided phase shift by the unit cell, shown in Figure 3.

Once the synthesis is finished and the required phase distribution is obtained, the patch lengths are adjusted element by element using the Method of Moments (MoM) [8] and a zero-finding routine. In this process, the real angle of incidence from the feed to each element of the reflectarray is used, in order to take into account possible phase shifts due to the incidence angle. Also, the MoM employs the Floquet theorem to analyze the unit cell, which embeds the analyzed element within an infinite array comprised of the same element, taking into account mutual coupling and the reflected field from the ground. It is thus important that the patch size variation is smooth in order to fulfill the local periodicity assumption of the analysis technique. Also, the design produces slightly rectangular patches, since the reflection coefficients are to some extent different for both polarizations. 


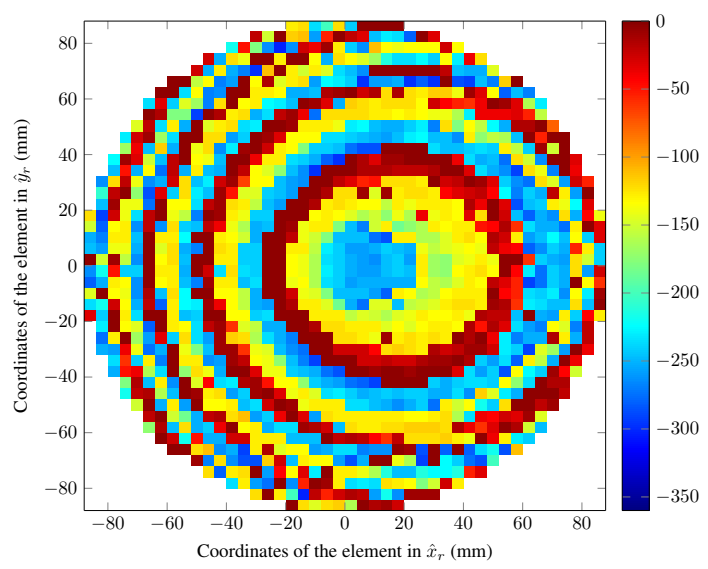

Figure 4: Synthesized phases for an isoflux pattern in X polarization with a phase restriction of $300^{\circ}$. Phases for Y polarization are very similar.

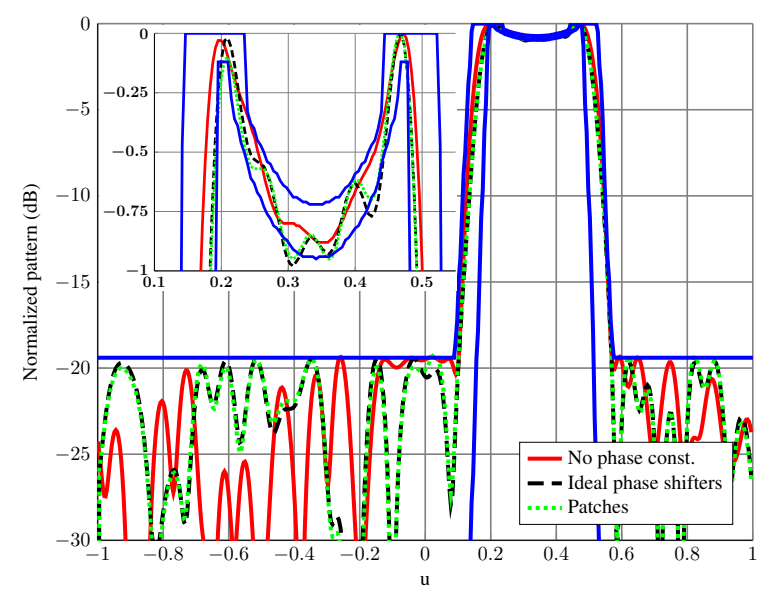

Figure 5: Main cut for $v=0$ of the synthesized isoflux pattern for three different simulations for $\mathrm{X}$ polarization: reference simulation (no phase constraints, red solid), simulation with ideal phase shifters (phase constraints, dashed black) and design simulation (phase constraints, dotted green).

\section{REsults}

The synthesis was carried out for both polarizations, obtaining a dual-linear polarized reflectarray with an isoflux pattern in both polarizations. The results for both polarizations are similar, and only those for the X polarization are shown. Figure 4 shows the synthesized phases with a total phase range of $300^{\circ}$ imposed during the synthesis process. As reference, a synthesis with no phase constraints was also performed.

Figure 5 shows the results between the synthesis with and without phase constraints. When the phase is free to vary in the whole $360^{\circ}$ range, a smooth coverage area can be obtained. However, restricting the phase range to $300^{\circ}$ causes the algorithm not to converge properly, an there is a ripple in the coverage zone. It is also shown the simulation after the design was made, simulating the patches with MoM [8], and there is little variation in the copolar component with regard to the simulation with ideal phase shifters.

After the phase-only synthesis, the reflectarray was manufactured and measured. Figure 6 shows a picture of the prototype with the supporting structure, which secures the position of the feed and the reflectarray breadboard. The antenna was measured in a spherical far-field range anechoic chamber at $30 \mathrm{GHz}$ and the measurements are shown in Figures 7 and 8 . The deterioration in the coverage area is attributed to several causes. First, in the synthesis process the corners of the reflectarray were not taken into account. Also, the coverage area is very sensitive to variations in the height and relative permittivity of the substrate. New simulations were performed for a square reflectarray and different values of the parameters of the substrate. A better agreement between simulations and measurements was obtained than with the circular reflectarray (see Figure 8). Side lobes are also affected by reflections in the supporting structure and diffraction in the edge of the breadboard, which were not taken into account.

These issues can be minimized by choosing a more appropriate antenna geometry, which reduces the interference of the supporting structure, and by employing a rectangular reflectarray instead of a circular one, having more variables to adjust the 


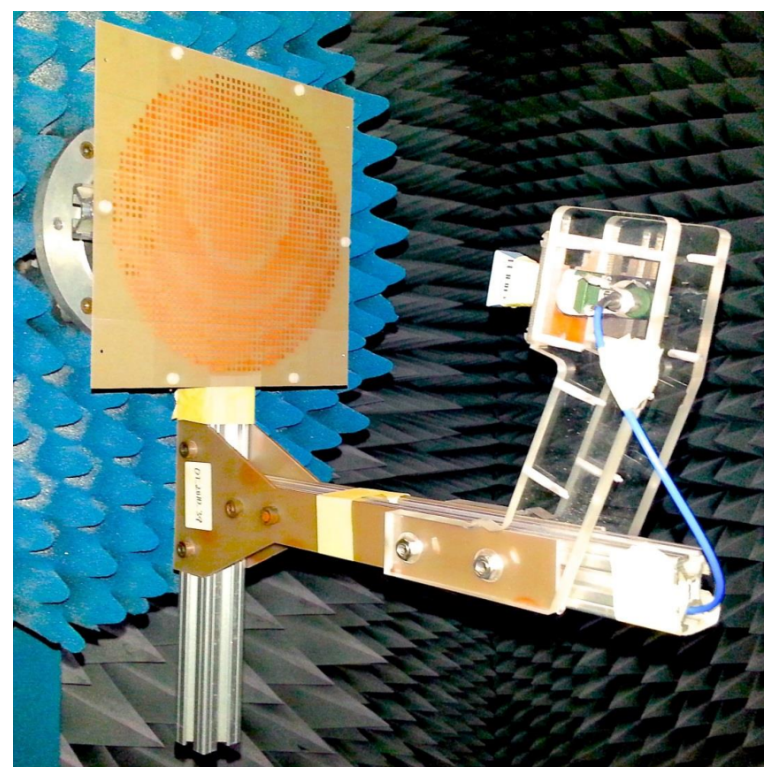

Figure 6: Picture of the prototype in the anechoic chamber.

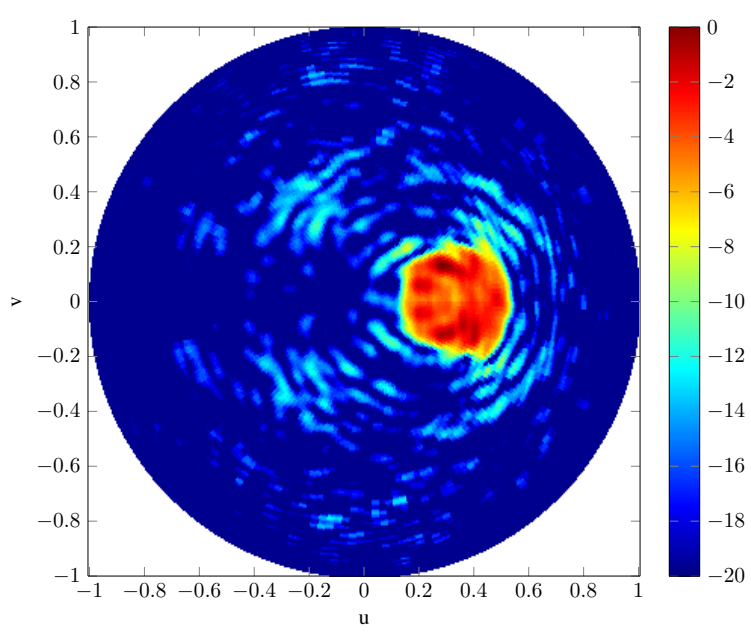

Figure 7: 3D measured pattern for $\mathrm{X}$ polarization.

pattern and taking into account the corners of the breadboard. Also, decreasing the illumination at the edge would reduced the diffraction effects.

\section{CONCLusion}

A low-cost reflectarray for global Earth coverage with isoflux pattern has been designed, manufactured and measured. A detailed development is presented to obtain an isoflux template for array synthesis for a generic satellite, which is later used in a reflectarray phase-only synthesis. The chosen element for the reflectarray is a single patch backed by a ground plane, which is the simplest element available. However, it is not able to provide a full $360^{\circ}$ phase range. For that reason, the Intersection Approach algorithm is used enforcing a phase restriction to match the range provided by the unit cell. After the synthesis process is finished, the required phase shift in each element of the reflectarray is obtained. The phase restriction causes the coverage area to have a higher ripple than if there was no phase restriction. A prototype was manufactured and measured. The degradation in the coverage area is caused by the corners of the reflectarray, not taken into account in the synthesis process, and variations in the height and permittivity of the substrate. Side lobes are affected by the suporting structure reflections and diffractions at the edge of the breadbord. These issues can be minimized by a proper choice of the whole antenna geometry and by considering a rectangular reflectarray instead of a circular one.

\section{REFERENCES}

[1] J. Huang and J. A. Encinar, Reflectarray Antennas. John Wiley \& Sons, 2008. 

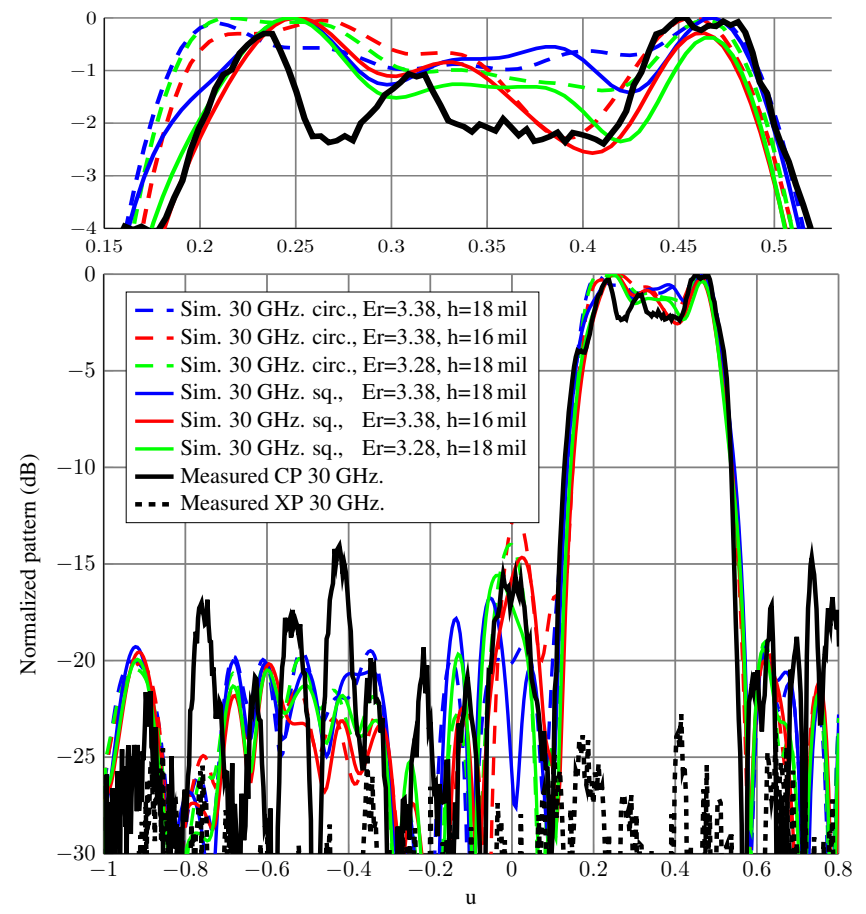

Figure 8: Measurement and simulations for $v=0$ for $\mathrm{X}$ polarization.

[2] J. A. Encinar and J. A. Zornoza, "Broadband design of three-layer printed reflectarrays," IEEE Trans. Antennas Propag., vol. 51, no. 7, pp. 1662-1664, Jul. 2003.

[3] L. Moustafa, R. Gillard, F. Peris, R. Loison, H. Legay, and E. Girard, "The phoenix cell: A new reflectarray cell with large bandwidth and rebirth capabilities," IEEE Antennas Wireless Propag. Lett., vol. 10, pp. 71-74, 2011.

[4] J. A. Zornoza and J. A. Encinar, "Efficient phase-only synthesis of contoured-beam patterns for very large reflectarrays," Int. J. RF Microw. Comput. Eng., vol. 14, no. 5, pp. 415-423, Sep. 2004.

[5] C. A. Balanis, Ed., Modern antenna handbook. John Wiley \& Sons, 2008.

[6] Y.-T. Lo and S.-W. Lee, Eds., Antenna Handbook Vol. 1. Van Nostrand Reinhold, 1993, ch. 1, pp. 28-29.

[7] S. D. Targonski and D. M. Pozar, "Analysis and design of a microstrip reflectarray using patches of variable size," in Antennas and Propagation Society International Symposium, vol. 3, Seattle, Washington, USA, Jun. 20-24, 1994, pp. 1820-1823.

[8] C. Wan and J. A. Encinar, "Efficient computation of generalized scattering matrix for analyzing multilayered periodic structures," IEEE Trans. Antennas Propag., vol. 43, no. 11, pp. 1233-1242, Nov. 1995. 